\title{
Cross Correlation Studies in Primate Motor Cortex: Synaptic Interaction and Shared Input
}

\author{
John T. Murphy, Hon C. Kwan and Yiu C. Wong
}

\begin{abstract}
Awake, unrestrained monkeys were trained to reach out with the forelimb and touch a button. Extracellular spike trains were recorded from pairs of neurons in contralateral precentral cortex with the same or separate microelectrodes. The neurons were located in the same or different functional columns as defined by intracortical microstimulation and passive sensory stimulation. Cross correlation analysis showed patterns consistent with synaptic excitation and/or inhibition between members of the cell pairs during the voluntary movement. The strength of correlation was inversely related to distance between columns, with the strongest correlations found between cells within the same column. Inhibitory correlations were virtually restricted to cell pairs within a single column. Temporal analysis showed that direct synaptic interaction and shared input patterns could be clearly distinguished in this physiologic setting. Spatial analysis indicated that shared input was concentrated among columns in the same and adjacent joint controlling zones as well as within a single column. No directional preference of shared input was present, a finding which was consistent with the observed nested organization of the forelimb area.
\end{abstract}

RÉSUMÉ: Études de corrélation croisée dans le cortex moteur du singe: L'interaction synaptique et l'entrée partagée. Des singe éveillés sans contraint ont été entraînés d'atteindre avec l'avant-bras et de toucher un bouton. Des trains de pointes extracellulaires ont été enregistrés de paires de neurones dans le cortex précentral contralatéral en employant les mêmes ou autres microélectrodes separées. Les neurones se trouvèrent dans les mêmes ou différentes colonnes fonctionelles tel que définies par la microstimulation intracorticale et par la stimulation sensorielle passive. Une analyse de corrélation croisée a démontré une formule en accord avec l'excitation synaptique et/ou l'inhibition entre les deux membres de la paire de cellules pendant le mouvement volontaire. Le degré de corrélation était inverse en relation avec la distance entre les colonnes, les corrélations plus fortes étant entre des cellules dans la même colonne. Des corrélations inhibitoires étaient presque limitées à des paires de cellules dans une colonne unique. L'analyse temporale démontre que l'interaction synaptique directe et des formules d'entrée partagées pouvaient être clairement distinguées dans ce milieu physiologique. L'analyse spatiale indiquait que l'entrée partagée était concentrée parmi des colonnes dans les mêmes zones et des zones de contrôles d'articulations adjacentes, ainsi que dans une colonne unique. Aucune direction préférentielle de cette entrée partagée ne fut presentée, une résultat en accord avec l'organisation en anneaux nidiformes de l'aire avant-bras du cortex cérébral.

Can. J. Neurol. Sci. $1985 ; 12: 11-23$

In previous studies of primate precentral cortex, we showed the presence of multiple groupings of neurons arranged in irregularly shaped "columns" orthogonal to the cortical surface (Kwan et al., 1978; Murphy et al., 1978; Wong et al., 1978). These columns display tight input-output coupling in a way which allows control of limb position about individual joints (Murphy et al., 1978). Columns controlling limb position about particular joints are themselves arranged in concentric nested and overlapping zones, with distal joints represented at the centre. Later studies indicated that neurons in these columns participate in voluntary movements about the appropriate forelimb joints (Wong et al., 1980; Kwan et al., 1981; Murphy et al., 1982 a,b).
In the present research, we address the questions of functional interactions and shared input among cells in the same or different columns in the context of a voluntary movement in awake monkeys. Anatomic studies have shown evidence of monosynaptic connections between neurons within precentral cortex (Jones and Wise, 1977; Jones et al., 1978; Gatter and Powell, 1978). Physiologic evidence of such connections is quite scarce (Asanuma and Rosen, 1973). Of particular note is the study of Allum and co-workers (1982) who found evidence of correlation between two neurons extracellularly recorded with the same electrode. Such neurons probably reside within the same functional column.

Anatomic evidence also indicates that several columns might 
share input from a common source (Jones and Wise, 1977; Jones et al., 1978, 1979; Szentagothai, 1978). For example, the axons of thalamic neurons are known to branch widely within motor cortex (Strick, 1976a; Jones, 1983). Physiologic assessment of shared input in motor cortex has not previously been undertaken.

In the present study, we employ the technique of cross correlation analysis of the firing patterns of pairs of separate functionally identified neurons in motor cortex. The activities of the neurons are recorded simultaneously from one or two electrodes as a monkey makes a natural reaching movement. The data provide information in a physiologic context on the nature and degree of synaptic interaction and shared input between cells located in the same and separate functional columns.

\section{METHODS}

Experiments were carried out on two Macaque monkeys trained to reach out with the right forelimb to touch a button, resulting in a fruit juice reward (Murphy et al., 1982b). Lighting of the button served as the signal to the monkey to initiate the task from a resting position. The onset of movement was recorded by the use of a microswitch which was activated when the monkey's arm left the support provided in the resting position. Following training, animals were prepared for chronic daily recording sessions by techniques previously described (Wong, et al., 1978). A custom-made device was utilized permitting simultaneous placement of two electrodes within a chamber overlying the forelimb area of contralateral precentral cortex (Kwan et al., 1980). Cells were identified with respect to their peripheral connections by means of passive sensory stimulation (Wong et al., 1978) and intracortical microstimulation (ICMS), (Kwan et al., 1978). All cells so identified were found to be functionally related to forelimb joints. Columns are operationally defined by the effects of ICMS at the recording site (i.e. "elbow flexion column'). For brevity, the output function of a cell is defined as the same as that of the column in which the cell resides. Justification for these operational definitions has been provided in previous experiments (Murphy et al., 1978, 1982 a,b). Recording sites were identified by histologic reconstruction using methods previously described (Wong et al., 1978). Distance between cells was measured in the horizontal plane.

The activities of two cells recorded from either the same or a different electrode were recorded simultaneously. Each electrode could be manipulated independently. The timing relationship of each spike train was assessed using an all-order cross-correlogram (Perkel et al., 1967). Data were collected during the stimulusbehavioural response paradigm. All cells were studied using both 1 and $5 \mathrm{~ms}$ bin correlograms. A frame shuffling procedure was carried out to assess correlation due to the triggering stimulus or behavioural event (Dickson and Gerstein, 1974, Murphy et al., 1985a).

To determine the presence of a significant correlation in the 5 $\mathrm{ms}$ bin gross correlograms, $\mathrm{z}$ values ( $\mathrm{t}$ test) were generated and the central hill (peak) compared to background. A p value of $<.01$ was used as a minimum significance level. In addition, autocorrelograms of each cell were constructed to ensure that the peaks of significant correlation were not due to fluctuations in the average firing probability of either of the cells involved.

To determine the presence of a significant correlation in the 1 ms bin gross correlograms, the Sears and Stagg criterion (1976) was applied. A ratio, $k$ (peak bin count/mean bin count), was computed and a $p<.01$ confidence level was chosen as the minimum significance level. In addition, $1 \mathrm{~ms}$ bin autocorrelograms of each cell were constructed to ensure that the peak or valley of the significant correlation was not due to fluctuations in the average firing probability of either of the cells involved.

Subtraction of the frame shuffled from the gross correlogram produces a difference correlogram. The presence of a peak or valley in the difference correlogram may be interpreted as indicating either direct, i.e. synaptic, interaction between two neurons, or a common source of input to each member of the pair. These conclusions have been based on simulation studies, and experiments in invertebrates and mammalian cerebral cortex (Perkel et al., 1967; Moore et al., 1970; Dickson and Gerstein, 1974; Tatton and Sokolove, 1975; Michalski et al., 1983). Two major features serve to distinguish the two possibilities. The first is the duration of significant interaction. Synaptic interactions tend to produce short duration, i.e. less than $10 \mathrm{~ms}$, changes in the difference plots. As a corollary, the slope of the observed peaks or valleys are quite sharp. A second feature is that of symmetry of the shoulders of the pattern. Synaptic interactions tend to have asymmetric patterns, with one shoulder predominating. Both these observations are true for either peaks or valleys. In contrast, shared input networks tend to produce correlation patterns which are of longer duration and symmetric.

\section{RESULTS}

\section{Synaptic Interaction}

Representative raw data in the form of spike trains and results of analysis are presented for a pair of neurons recorded from the same electrode (Fig. 1). These neurons were situated within a column identified by ICMS as causing internal rotation of the arm at the shoulder. Figure lA shows four of twenty three trials during the stimulus-response paradigm, with the two spike trains recorded simultaneously. In Figure IB, autocorrelograms are plotted for each cell. These are analysed and plotted using $1 \mathrm{~ms}$ bins, and show no periodicity for either cell which might contribute to the observed cross correlations. The slightly higher background firing frequency of cell $A$ in comparison to cell $B$ is evident.

Cross correlograms for this cell pair are shown in Figure 1C. The gross correlogram shows forward and backward renewal densities, of all orders. The dotted line indicates the mean number of counts per bin, which shows how often an interval might occur if cell A and cell B were firing independently. This measure is sometimes called the "reference level" (Perkel et al., 1967) or "baseline" (Mastronade, 1983) of the cross correlogram. The k-ratio test as defined in Methods indicates a significant correlation at -2 to $0 \mathrm{~ms}$, as evidenced by the sharply defined peak in the correlogram. Shuffling the cross correlogram provides a measure of event related correlation, and none is present in this instance (Fig. 1C, middle). The difference between the gross and shuffled cross correlogram (Fig. 1C, lower) indicates synaptic interaction between the two cells (Perkel et al., 1967).

Figure $2 \mathrm{~A}-\mathrm{C}$ shows another pattern of synaptic interaction in the cross correlogram, this being a combination of valleys and peaks. In this instance, the two neurons, again recorded from a single electrode, were functionally related to external rotation 
A

$\begin{array}{cccc}\downarrow & m & m m & m \\ \begin{array}{c}\text { STIMULUS } \\ \text { ONSET }\end{array} & \begin{array}{c}\text { MOVEMENT } \\ \text { ONSET }\end{array} & \begin{array}{c}\text { TOUCHING } \\ \text { BUTTON }\end{array} & \text { RETURN } \\ & & \end{array}$
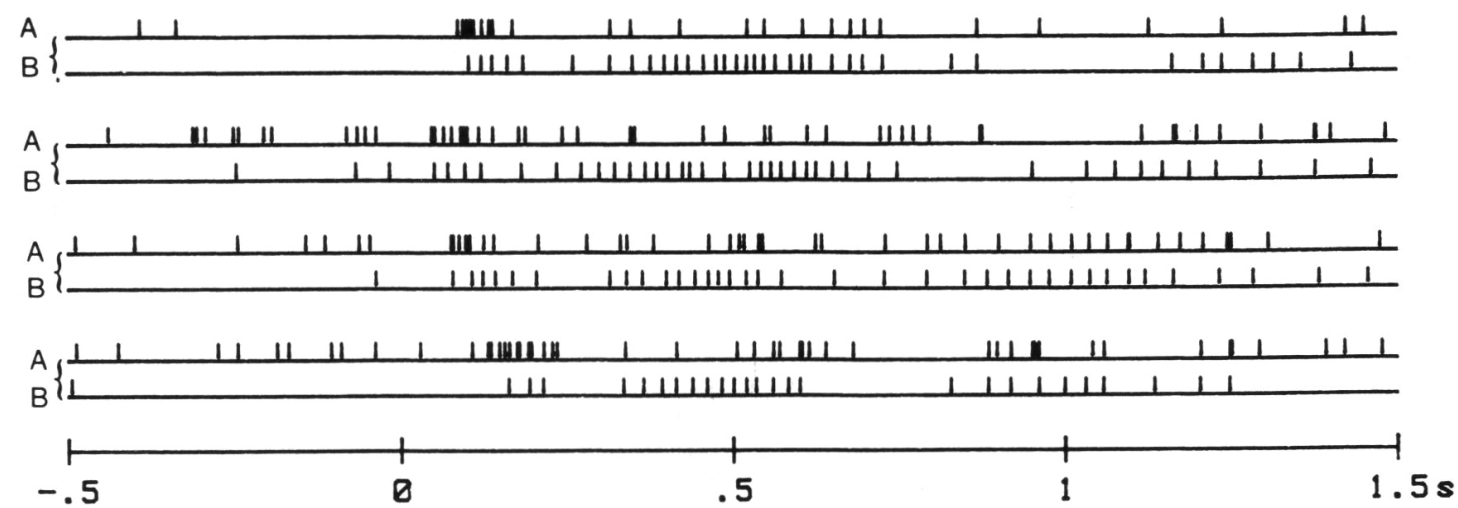

B RUTOCORRELOGRAMS

Cell A

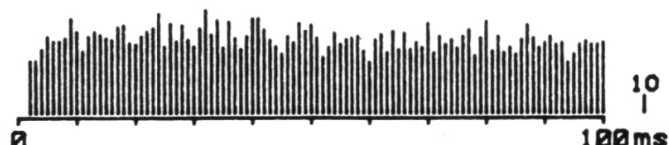

$100 \mathrm{~ms}$

Cell B

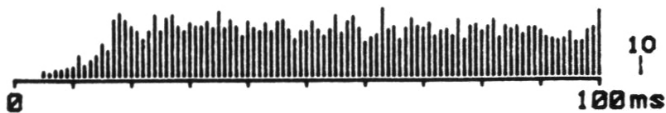

C CROSS CORRELOGRams A $\rightarrow$ B

GROSS

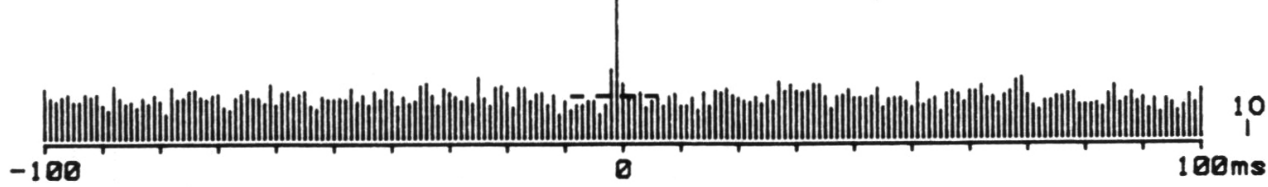

SHUFFLED

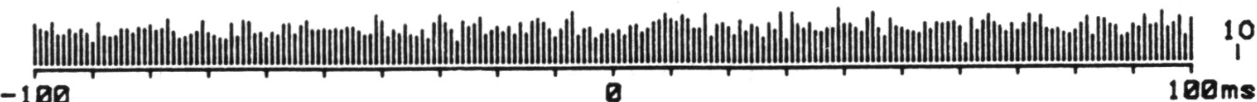

DIFFERENCE

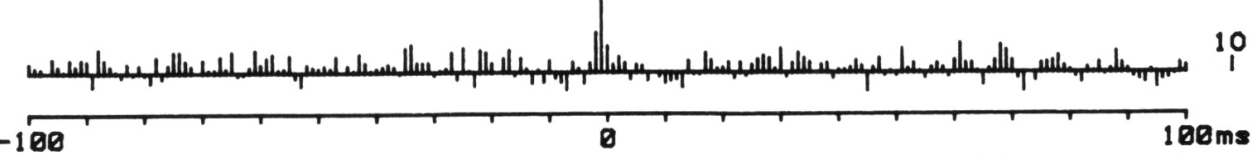

Figure $l-A)$ Spike trains of two shoulder internal rotation cells recorded simultaneously from I electrode during the reaching task. The arrow indicates onset of the visual stimulus, $500 \mathrm{~ms}$ after the start of each trial. Three sets of vertical bars represent the range of times when the monkey lifts his arm from the resting p!ate, touches the button and returns his arm to the control plate, respectively.

$B) I \mathrm{~ms}$ bin autocorrelograms of cells $A$ and $B$, respectively. To the right of each plot is a scale indicating the number of counts per bin.

C) $1 \mathrm{~ms}$ bin cross-correlograms of cells $A$ and $B$ derived from data of 23 trials. $k$-ratio (at -1 ms bin) is 7.5 , mean bin count $=25$, hence $p<.001$. Frame shuffing is used to generate the "shuffled" cross correlograms. The above conventions also apply to Figure 2. 


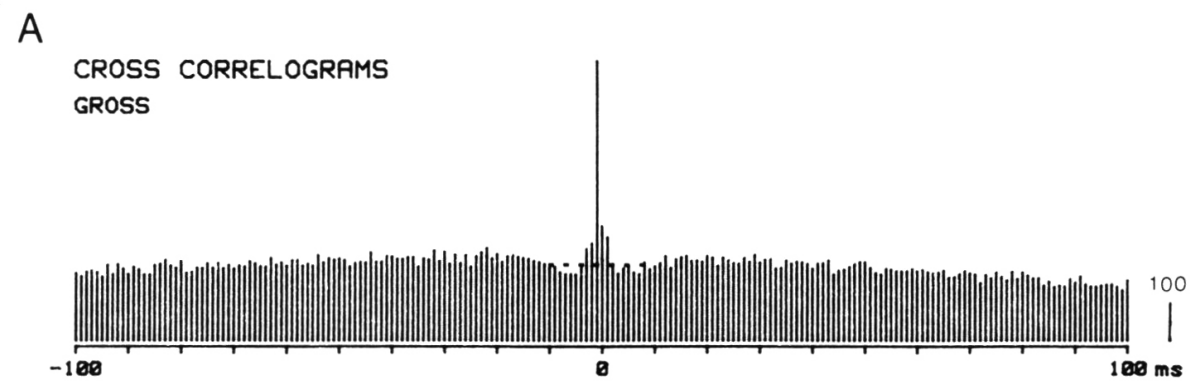

B

SHUFFLED

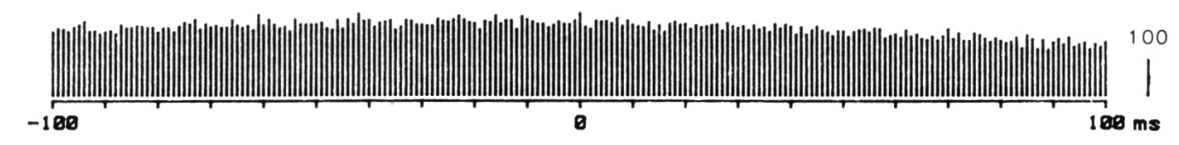

C

DIFFERENCE

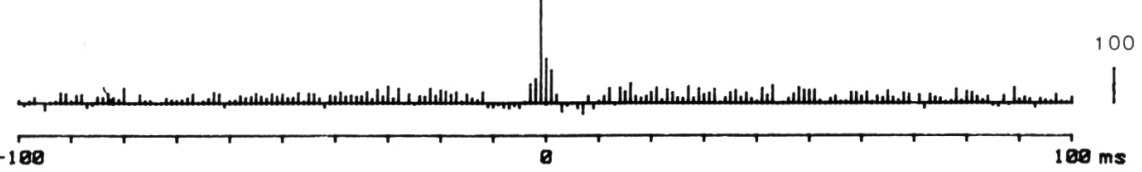

D

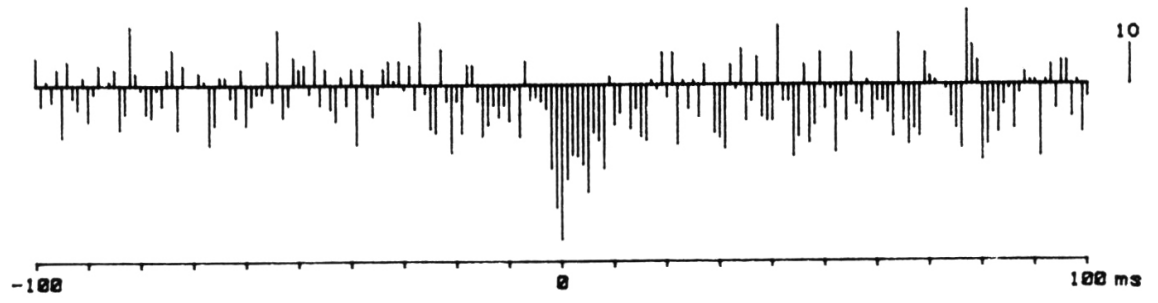

$E$

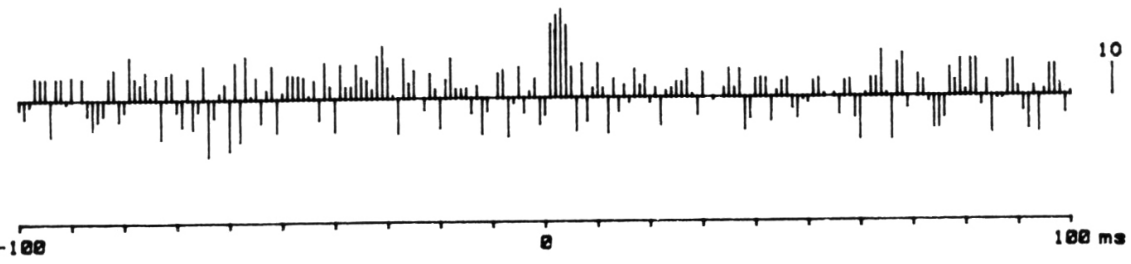

Figure $2-A-C)$ Cross correlograms of two elbow extension cells recorded from the same electrode. $k$-ratio $($ at $0 \mathrm{~ms}$ bin $)=1.8$. Mean bin count $=122, p<.001$. D) Difference correlograms of two shoulder external rotation cells recorded from the same electrode. $k$-ratio $(a t-1$ ms bin) $=3.75$, mean bin count $=23$, $p<.001$.

E) Difference correlograms of an elbow flexion cell and a wrist supination cell recorded from two different electrodes, $k$-ratio (at $3 \mathrm{~ms})=1.34$, mean bin count $=61, p<.01$. 
of the shoulder. No event-related correlation was demonstrated by the shuffled correlogram. The difference plot shows a complex pattern, with a valley-peak-valley complex ranging from about -10 to +10 msec.

A third type of synaptic interaction is shown in Figure 2D. Here a valley alone is present in the difference plot. Again, the shuffled correlogram (not illustrated) showed no evidence of event-related correlation. The two neurons were recorded from the same electrode, and were each functionally related to elbow extension. Significant levels in the valley range from about -2 to $9 \mathrm{msec}$, as seen in the difference plot.

Cell pairs recorded from two electrodes showed in general weaker synaptic correlations than pairs recorded from a single electrode. An example is shown in Figure 2E. The two neurons were functionally related to elbow flexion and wrist supination, respectively. The difference plot showed a significant peak from 1 to $4 \mathrm{msec}$. Table 1 shows a comparison of significance of correlation between pairs of cells recorded from one or two electrodes, with significance based on k ratio tests. Of the 237 pairs, about one third were derived from one electrode and two thirds from two electrodes. The majority of cell pairs recorded from one electrode showed $\mathbf{k}$ ratios with $p$ values of less than .001 , while none of the pairs recorded from two electrodes showed this degree of significance. Table 1 also shows a gradual decrement in percentage of correlated pairs with increased distances between the two cells of the pair.

In addition to differences in degree of significance, the two groups of pairs also showed differences in the presence of significant synaptic interaction. Fifty one percent of pairs recorded from a single electrode showed correlations at p levels less than .01 , while $26 \%$ of pairs recorded from two electrodes showed correlations at this significance level (Table 1). The probability of finding any correlation among all pairs was $35 \%$. It is of interest that of the 82 pairs which showed significant synaptic interaction, slightly less than half (39) showed this pattern alone. In the remainder, various mixtures of synaptic interaction with shared input and/or event related (Murphy et al., 1985a) patterns were observed.

The timing during which significant synaptic interaction existed also differed between pairs recorded from one or two electrodes. As shown in Figure 3, pairs recorded from one electrode tended to have short durations of correlation, clustered about time \pm 1 ms. Temporal correlation patterns for cells showing simple valleys peaks or complex patterns are illustrated in this figure. Pairs recorded from two different electrodes also showed short correlation durations, the majority being less than $5 \mathrm{~ms}$. However, in contrast to the one electrode group, times of correlation were centered away from time 0 for these pairs, as shown. Another difference between the two groups was the almost exclusive occurrence of peaks rather than vallèys in the correlations recorded between two electrodes.

\section{Shared Input}

Figure 4A shows peri-stimulus time histograms for 2 cells recorded from the same electrode. ICMS at the electrode site produced external rotation of the shoulder. Both cells showed coincidence of firing with the reaching task. The autocorrelograms show no periodicity for either cell (Fig. 4B). Gross, shuffled and difference correlograms are shown in Figure 4C. Correlation related to the event (reaching task) is observed in the shuffled correlogram (Murphy et al., 1985a). Broad, sloping shoulders and a relatively long duration of significant correlation are seen in the difference plot, and these are characteristic of shared input correlation (Methods). In addition, a sharp peak typical of direct synaptic interaction is superimposed in this plot. A total of 57 of 237 pairs (24\%) showed shared input. Of these 57,10 showed correlation patterns typical of only shared input, while the remainder showed various combinations of shared input with synaptic interaction, as in the illustration, and/or event related correlation. Table 2 summarizes the incidence of shared input in all pairs.

The cell pair in Figure 5A shows no event-related correlation. The upward trend in the shuffled data is due to the firing pattern of the individual cells and does not indicate functional interaction. These cells were recorded by one electrode. Both were located in the same column, and the latter produced internal rotation of the shoulder on ICMS at the recording site. A combination of shared input and synaptic interaction is also observed in the difference plot.

Figure 5B shows data from a pair of cells recorded with 2 separate electrodes. These cells were identified by ICMS as belonging to wrist ulnar deviation and finger extension columns respectively. No event related correlation is present in the shuffled plot. The difference plot shows essentially a pure shared input interaction, shifted to the right of the origin. At other times, shared input interactions were shifted to the left of the origin, as in Figure 5A.

"Valley" patterns compatible with shared input were recorded in $10 \%$ of the 57 correlated pairs. An example is shown in Figure $5 \mathrm{C}$. These cells, recorded from the same electrode, were members

Table 1: Frequency Distribution of Significantly Correlated Pairs Showing Synaptic Interaction at Various Distances Between Electrodes

\begin{tabular}{|c|c|c|c|c|c|c|}
\hline & Distance $(\mu)$ & $\mathrm{p}<.01$ & $\mathrm{p}<.005$ & $\mathrm{p}<.001$ & $\begin{array}{c}\text { Total } \\
\text { Correlated } \\
(\%) \\
\end{array}$ & $\begin{array}{c}\text { Total } \\
\text { Studied }\end{array}$ \\
\hline I Electrode & 0 & 6 & 3 & 33 & $42(51 \%)$ & 82 \\
\hline 2 Electrodes & 500 & 12 & 1 & 0 & 13 & 34 \\
\hline & 1000 & 16 & 1 & 0 & 17 & 54 \\
\hline & 1500 & 3 & 0 & 0 & 3 & 15 \\
\hline & 2000 & 6 & 0 & 0 & 6 & 33 \\
\hline & 2500 & 1 & 0 & 0 & 1 & 9 \\
\hline & $3000 /$ over & 0 & 0 & 0 & 0 & 10 \\
\hline TOTAL (2) & & 38 & 2 & 0 & $40(26 \%)$ & 155 \\
\hline TOTAL $(1+2)$ & & 44 & 5 & 33 & $82(35 \%)$ & 237 \\
\hline
\end{tabular}


A

ONE ELECTRODE

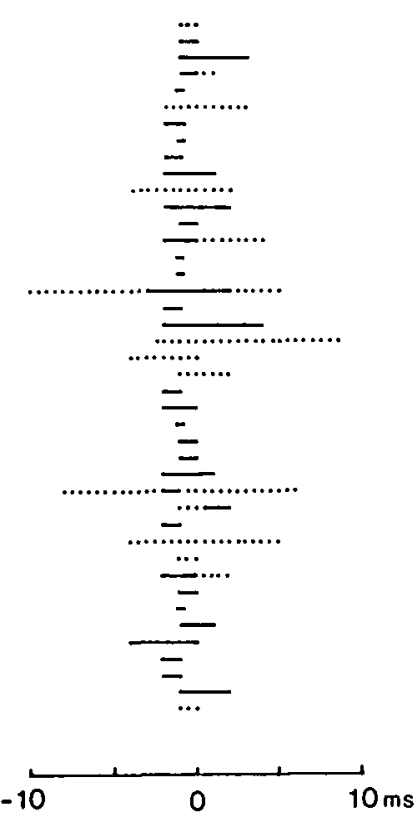

TIME OF CORRELATION
B

TWO ELECTRODES

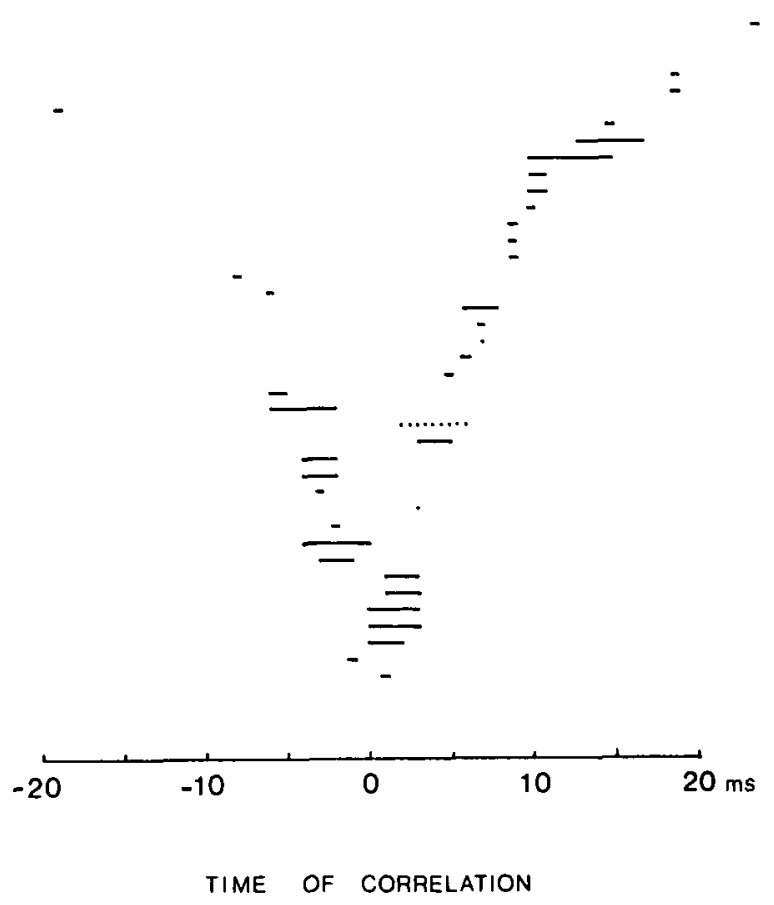

Figure 3 - Comparison of time of correlation between pairs of cells recorded from $I(A)$ and 2 electrodes $(B)$. Time of correlation is defined as the occurrence of a significant peak (solid line) or valley (dotted line) or combination of both in the difference correlogram.

of a column which produced ulnar deviation of the wrist on ICMS at the recording site. There is evidence of both shared input and synaptic interaction in the difference plot.

Fig. 5D shows a combined peak and valley shared input correlation. This type of correlation represents $14 \%$ of correlated pairs. These cells were recorded from the same electrode. ICMS at the recording site produced flexion about the wrist joint. There is also evidence of a synaptic interaction in this instance, as seen in the narrow peak superimposed on the broader "hill" pattern.

Several aspects of the spatial organization of shared input were also explored. The percentage of cell pairs showing shared input decreased linearly as a function of horizontal distance between cells. No shared input patterns were observed beyond $3 \mathrm{~mm}$. In contrast, strength of correlation as measured with the t-test was found to be independent of horizontal distance when pairs were recorded from two electrodes. Stronger correlations were infrequently seen in data recorded from one electrode.

Temporal characteristics of the shared input interactions are shown in Figure 6. The actual duration of significant correlation is shown for each cell pair in Figure 6A. Solid and dashed lines indicate peak and valley correlations respectively. A frequency histogram of all centroid times is shown in Figure 6B. Most of these times are non-zero. This would be expected in a situation where there were different transmission delays of shared input to each cell of the pair. Durations of significant shared input interactions are expected to be relatively long compared to those associated with synaptic interaction (Perkel et al., 1967), and comparison of Figures $6 \mathrm{~A}$ and $3 \mathrm{~A}$ shows this to be indeed the case in the present setting. Figure $6 \mathrm{C}$ shows a frequency histogram of durations of shared input interactions for all of the correlated cells of Figure $6 \mathrm{~A}$. This shows a range of $80-480 \mathrm{~ms}$ with a median value of $260 \mathrm{~ms}$.

In order to determine whether the observed shared input displayed a preferred directional orientation, a topographic map showing cell locations, as transposed to the cortical surface, was constructed. In Figure $7 \mathrm{~A}^{\circ}$, the loci of each cell of all correlated pairs is connected with a solid line. All uncorrelated pairs, represented by straight dashed lines, are superimposed. On inspection, neither the correlated nor the uncorrelated pairs showed any directional preference. The data is more syste-

Table 2: Distribution of Correlated Pairs Showing Shared Input Among Different Forelimb-Joint Zones

\begin{tabular}{|c|c|c|c|}
\hline & Studied & Correlated & $\%$ \\
\hline \multirow[t]{2}{*}{$\begin{array}{l}\text { Same jt (same electrode) } \\
\text { (diff. electrode) }\end{array}$} & $\begin{array}{l}82 \\
60 \\
\end{array}$ & $\begin{array}{l}27 \\
11\end{array}$ & $\begin{array}{l}32.9 \\
18.3 \\
\end{array}$ \\
\hline & 142 & 38 & 26.8 \\
\hline Adjacent jt & 70 & 17 & 24.4 \\
\hline Across $1 \mathrm{jt}$ & 15 & 1 & 7 \\
\hline \multirow[t]{2}{*}{ Across $2 \mathrm{jt}$} & 10 & 1 & 10 \\
\hline & $\overline{237}$ & 57 & 24 \\
\hline
\end{tabular}


A

$\underset{\text { Cell }}{\text { PSTH }} \quad$ o $\quad$ b $\quad$ r
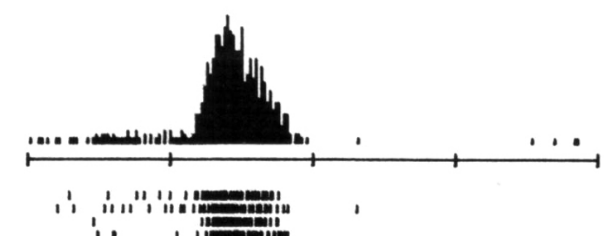

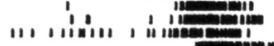

11

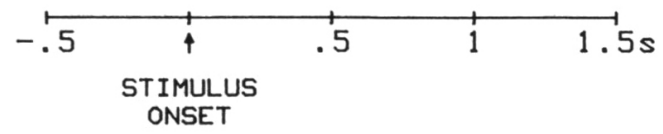

B

\section{AUTOCORRELOGRAMS}

Cell 1

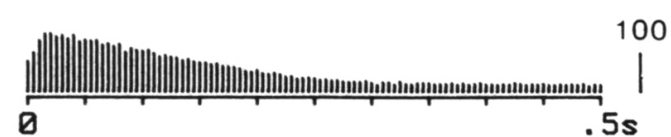

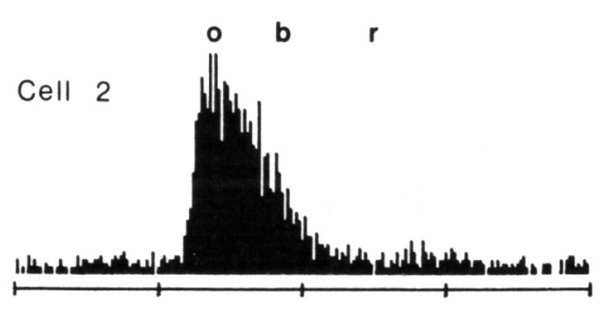
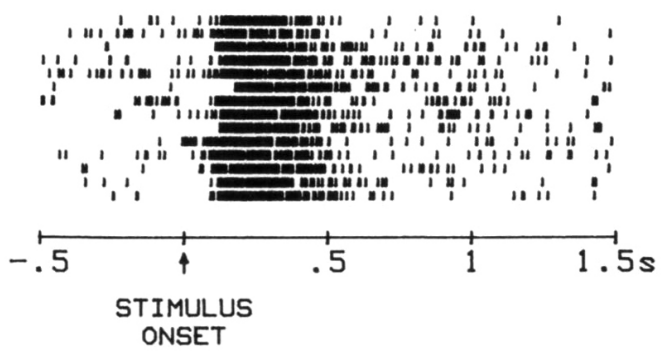

Cell 2

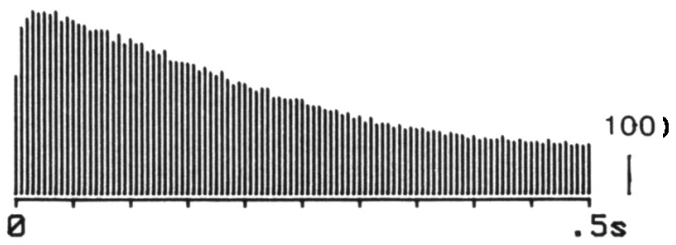

C cross correlograms $2 \rightarrow 1$
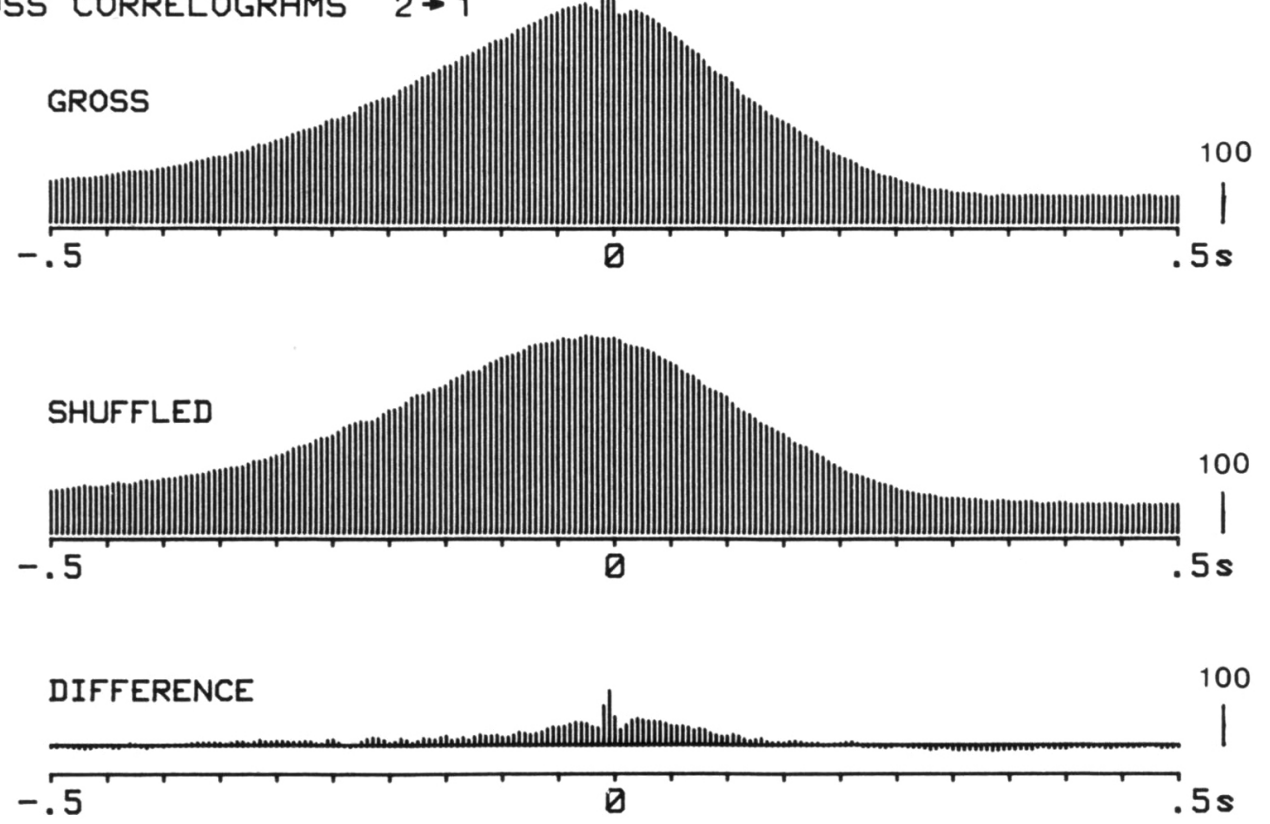

Figure 4 - A) Peristimulus histograms and raster plots (a total of 14 trials) of 2 shoulder external rotation cells recorded by one electrode. Each vertical bar in the raster represents an action potential.

B) 5-ms bin smoothed autocorrelograms of both cells. Vertical bar on the right indicates number of counts per bin as shown.

C) 5-ms bin smoothed cross-correlograms of cell 1 with respect to cell 2 . 
A

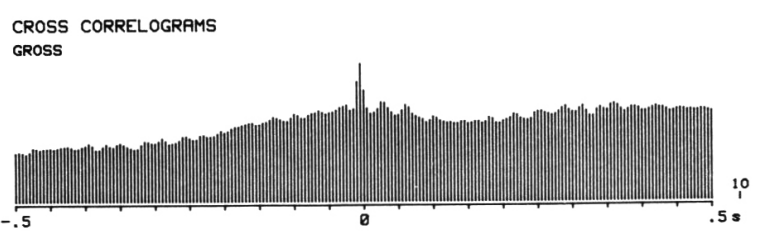

SHUFFLED

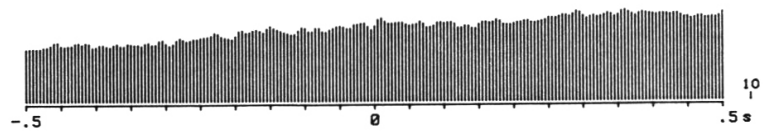

DIFFERENCE

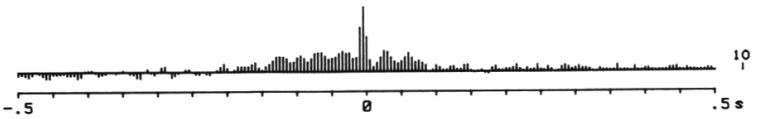

C

CROSS CORRELOGRAMS GROSS
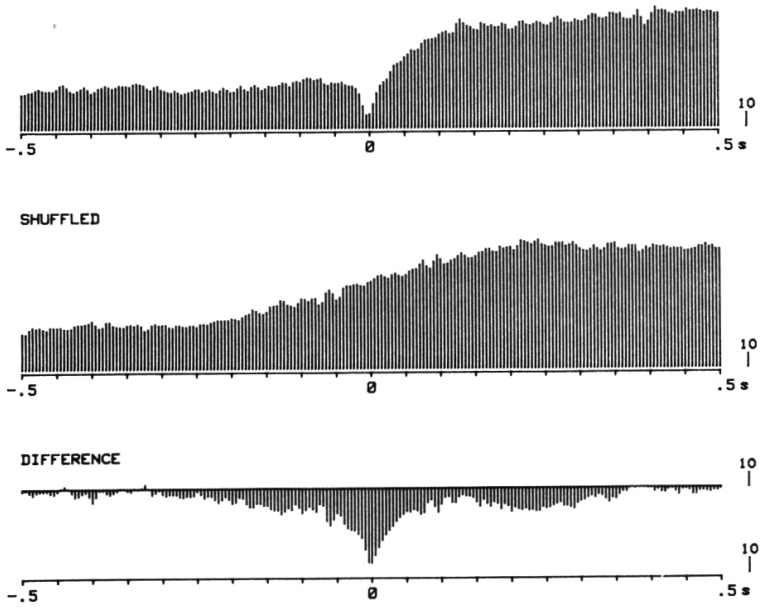

CROSS CORRELOGRAMS GROSS

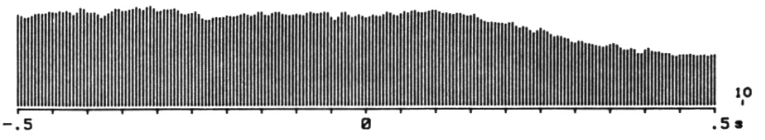

SHUFFLED

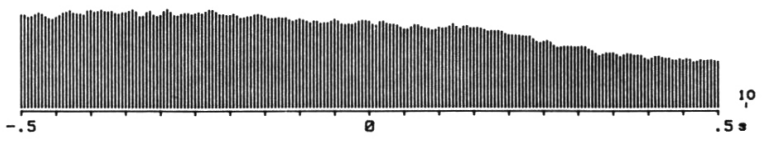

DIFFERENCE

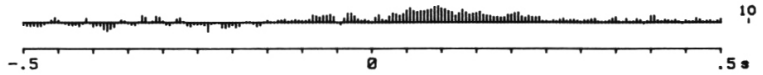

D

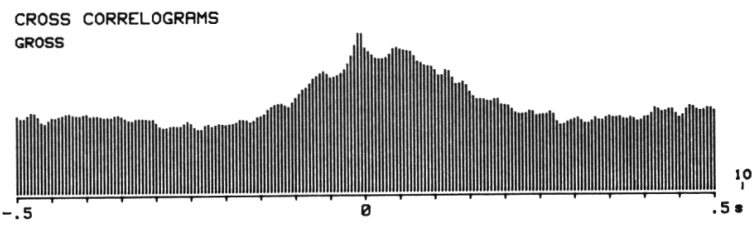

SHUFFLED

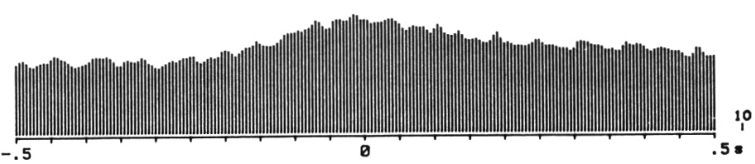

DIFFERENCE

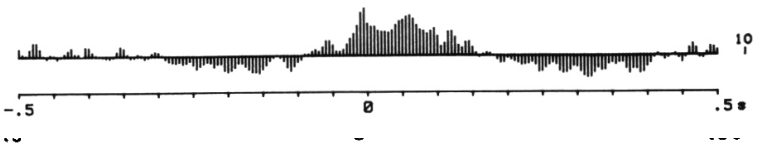

Figure 5-Cross-correlograms of 4 correlated pairs of cells. Conventions as in Figure 4.

A) 2 shoulder internal rotation cells recorded by l electrode.

B) A wrist ulnar deviation cell and a finger extension cell from 2 electrodes.

C) 2 wrist ulnar deviation cells recorded by 1 electrode.

D) Wrist flexion cells from 1 electrode.

matically presented in the polar frequency plot of Fig. 6B (bin width $45^{\circ}$ ). Again, shared input correlations are observed in all orientations.

To determine whether the shared inputs extended across nested zone boundaries, we identified the functional column for each cell in a cell pair. Results of this analysis are presented in Table 2. The data show that shared input correlation exists between columns controlling the same joint, and also between columns controlling adjacent joints. There is no significant difference at $\alpha=0.05$ ( $X^{2}$ goodness-of-fit test; Harnett, 1975) between percentages of correlated cells in these 2 cases ( $26 \%$ vs 24.4\%). In contrast, we observed shared input correlation very rarely between cells functionally coupled to non-adjacent joints.
It was possible to record up to 4 cells from the two electrodes, 2 from each. We observed shared input among 3 cells on two occasions, and among 4 cells on two occasions. These 4 examples were derived from a total of 43 instances in which 3 or more cells were analyzed. An example of shared input among 3 cells is shown in Figure 8. Here we present a joint impulse scatter diagram (Figure 8D) adapted from Perkel and colleagues (Perkel et al., 1975). The central density in the "snowflake" indicates input from a common source shared among 3 neurons. The associated difference correlograms for each combination of 2 cells are also illustrated (Fig. $8 \mathrm{~A}-\mathrm{C}$ ). To our knowledge, there is no previous published record of this pattern in primate brain. 
A
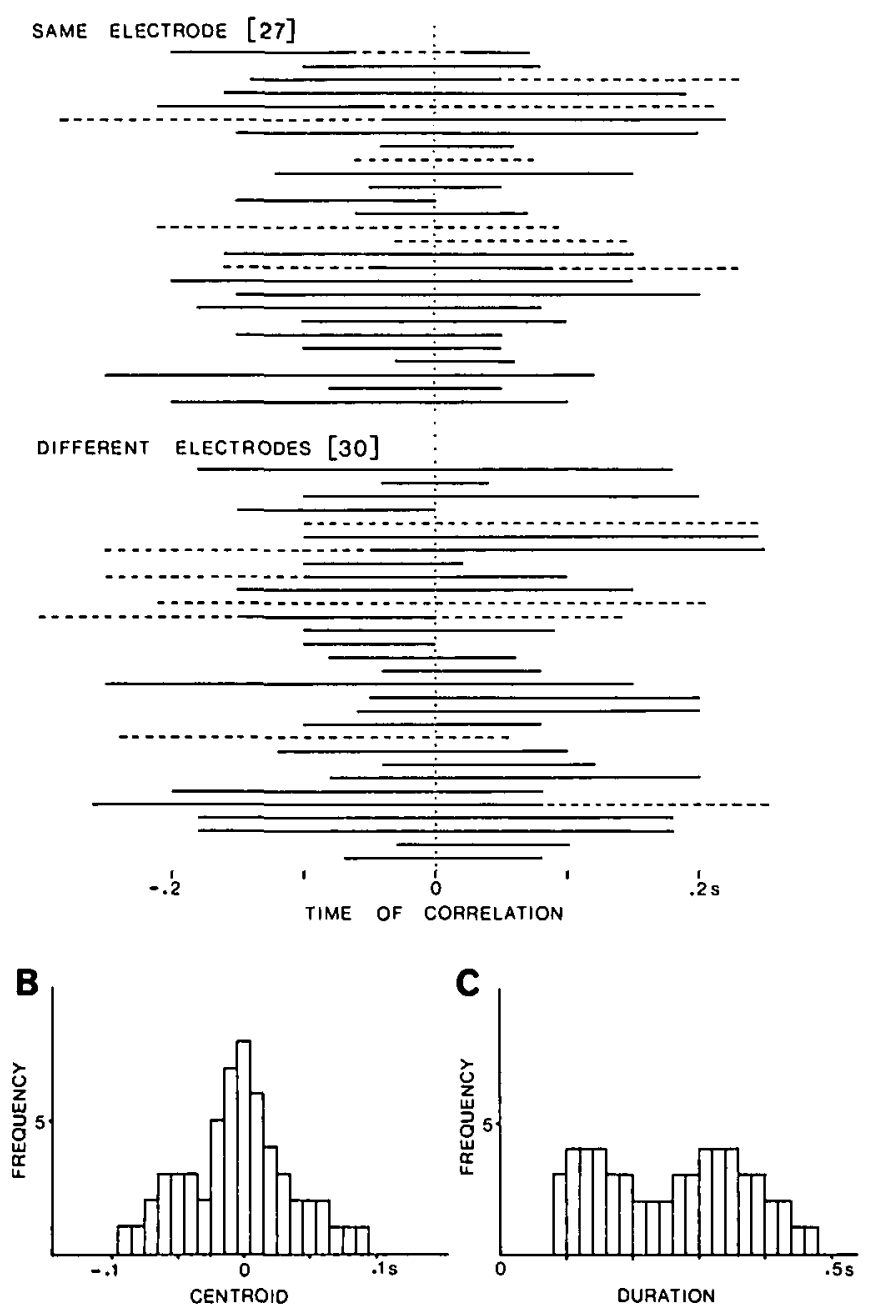

Figure 6-A)Time of significant correlation for each pair of correlated cells recorded by the same or different electrodes. A solid line represents a peak and dashed line a valley in the difference correlograms.

$B$ ) Frequency distribution of centroids of correlation using $10 \mathrm{~ms}$ bin size.

C) Frequency distribution of duration of correlation using $20 \mathrm{~ms}$ bin width.

\section{Discussion}

\section{Synaptic Interaction}

A total of 82 pairs in our data set meet the criteria for direct, synaptic interaction between neurons (Table 1). In 42 cases, various mixtures of synaptic interaction, shared input and eventrelated patterns (Murphy et al., 1985a) were observed. In the remaining 39 cases, only synaptic interaction was present.

When direct interaction is present, peaks imply synaptic excitation, and valleys inhibition (Perkel et al., 1967). The present study does not distinguish between mono-or polysynaptic connections. However, interactions occurring within $1 \mathrm{~ms}$ are probably monosynaptic, as judged by wave form morphology (Perkel et al., 1967; Moore et al., 1970; Knox, 1981; Allum et al., 1982), intracellular recording (Matsumura, 1979) and detailed analysis of invertebrate networks (Tatton and Sokolove, 1975). In our data (Fig. 5), interactions clustered about time $\pm 1 \mathrm{~ms}$ for cell pairs recorded from one electrode. The vast majority of cells in each pair recorded from one electrode arise from neurons within the same functional grouping of cells, and thus control movement about the same joint in a particular direction. The clustering of significant correlations about time less than $\pm 1 \mathrm{~ms}$ indicates that synaptic interactions among these cells may be mono- or oligo-synaptic.

When two cells of a pair were recorded from two different electrodes, these cells were of course separated by a greater distance from each other. Such cells were usually members of functionally different columns. These cells also showed synaptic interactions, but with a phase shift appropriate to the distance between cells (Fig. 3). In addition, when compared to pairs recorded from a single electrode, a smaller percentage of cells recorded from two electrodes showed significant correlation (Table 1). Moreover, when correlation was present in the latter cases, it was in general weaker (Table 1). Hence, the strongest synaptic interactions occur between cells close to each other, and such cells are usually controlling movement of a limb part about the same joint in a given direction (Figs. 1, 2 A-D). Nonetheless, significant although less potent, synaptic interactions are present between cells which control position of limb parts about either different forelimb joints (Fig. 2E), or the same joint but in different directions or planes of movement. The data recorded from two electrodes indicates that the number of correlated pairs falls off with distance between cell members (Table 1).

These physiologic findings correlate well with anatomic data which indicate that the density of monosynaptic connections in primate motor cortex is inversely proportional to distance between two points (Gatter and Powell, 1978; Phillips, 1981). The fact that significant correlations between cells recorded from two electrode pairs tend to be centered away from time 0 (Fig. 5) suggests that some of these synaptic interactions may be polysynaptic. Alternatively, the interactions might be subserved by monosynaptic paths of slow conduction velocity and/or low synaptic density or efficacy. As shown in Figures 1, 2 and $3 A$, excitatory, inhibitory and complex interactions were observed between cells recorded from one electrode. Undoubtedly, some of the cells are local interneurons, and others projection cells. Inhibitory interneurons are known to exist in motor cortex (Kelly and Renaud, 1974; Szentagothai, 1978), and these could contribute to the inhibitory and complex patterns observed. Interestingly, an inhibitory interaction was recorded in only 1 of 155 cell pairs recorded from two electrodes (Fig. 3). All of the remainder showed only excitatory interactions. This difference between the two data sets suggests that inhibitory interactions are found mainly within a single functional column, or at best between cells in immediately adjacent columns.

The results with a single electrode confirm those of Allum and co-workers (1982), and indicate synaptic interaction between cells in primate motor cortex during voluntary movements. It has previously been shown by spike-triggered averaging techniques that a majority of cells in primate motor cortex have muscle fields that include more than one synergistic muscle (Fetzand Cheney, 1978). The present data show one mechanism by which an enlarged muscle field may exist, namely by synaptic interaction between closely neighbouring cells in cortex. We also have provided, using two electrodes, the first demonstration of synaptic interaction among cells within different functional columns in motor cortex during a voluntary movement. This 


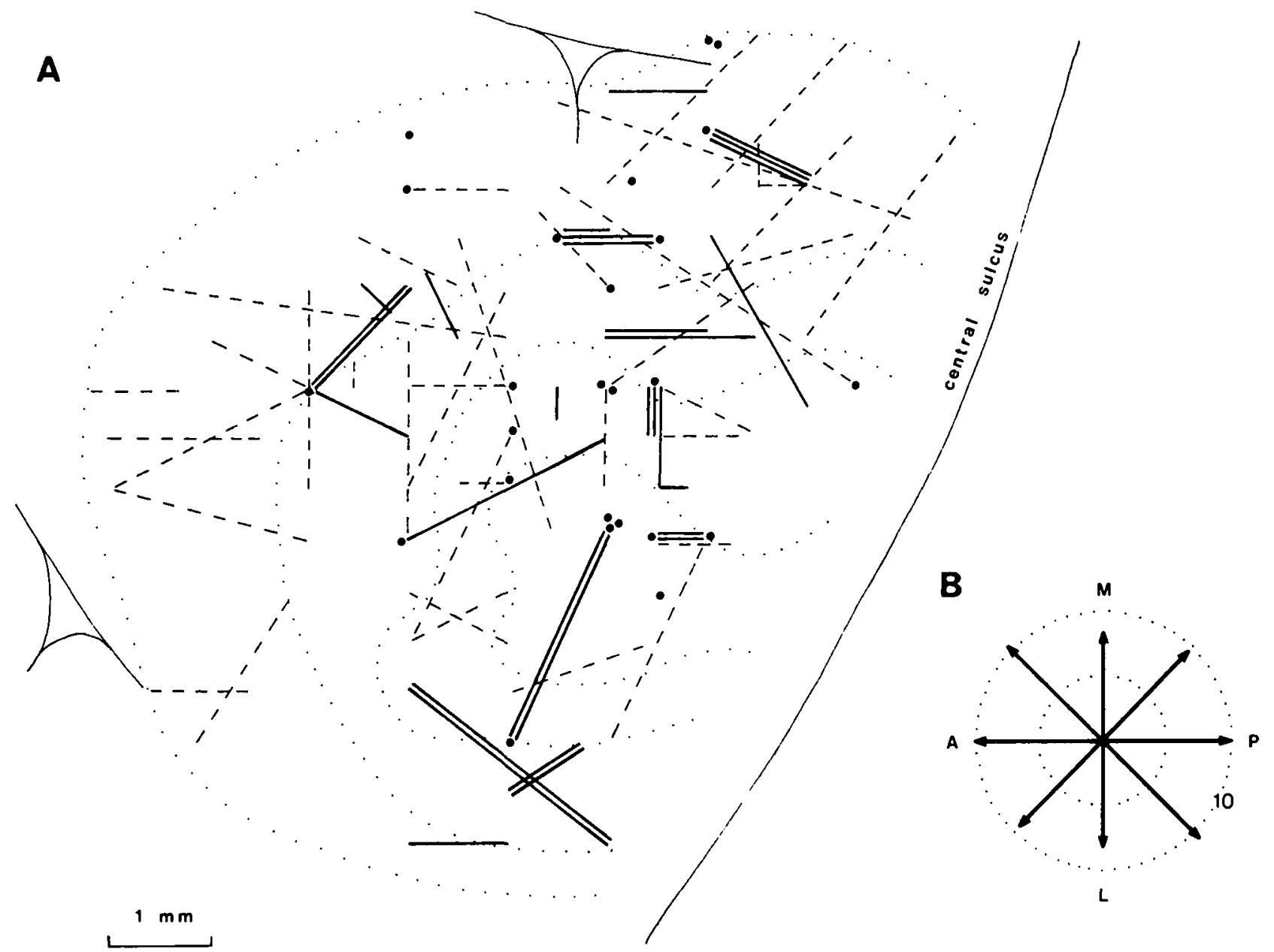

Figure 7 - A) Spatial locations of correlated pairs (solid line) and uncorrelated pairs (dashed lines) on the cortical surface. Each dashed line represents one or more pairs. Solid dots indicate locations of correlated pairs of cells recorded by same the electrode. Dotted lines show the boundaries of various identified forelimb joint zones: shoulder, elbow, wrist and fingers in an outer-to-inner order.

$B)$ Frequency distribution of correlated pairs of cells in a given orientation zone (45 sectors). Anterior (A)-Posterior (P): $20 \% ; M e d i a l(M)$-Laterial (L): $17.1 \%$; MP-AL: $19.4 \% ;$ MA-PL: $21.2 \%$. Percentage is measured by the length of the arrows (radius outer circle: $10 \%$ ).

data is compatible with that obtained in cat motor cortex using ICMS techniques (Asanuma and Rosen, 1973).

All of these results indicate that the columnar organization of motor cortex provides a physical substrate for information exchange. Moreover, the data reject any model of limb movement control which includes only parallel, independent output channels from motor cortex (cf. Murphy et al., 1985a). Our experiments show extensive communication among neurons within distinct columns contained in the nested zones of the forelimb area of precentral cortex. This communication operates during elaboration of a voluntary, reaching movement of the forelimb.

The finding of a strong synaptic correlation between cells in columns controlling the same or adjacent forelimb joints expands our understanding of how precentral cortex functions during such a reaching movement. Firstly, this synaptic interaction provides a portion of the basis for sequential activation of neuronal columns in a proximal to distal order, which has been observed in a reaching movement (Murphy et al., 1985 a,b). This in turn causes corresponding sequential EMG action (ibid;
Lamarre et al., 1981) and consequent sequential angular rotation of forelimb joints (Murphy et al., 1982b). A similarly tight kinematic coupling between contiguous forelimb joints has been observed in a human reaching movement (Soechting, 1984).

\section{Shared Input}

The data of Figures 4 and 5 show for the first time that shared input is present in primate motor cortex during a voluntary movement. This input is shared between cells within the same and different functional columns. We attempted to gather detail regarding the spatial arrangements of these shared inputs. The percentage of pairs showing a shared input decrements gradually with distance up to $3000 \mu$ and then abruptly drops off to 0 . This implies that axon branching from single sources should cover a distance of this magnitude, which is confirmed by anatomic studies (cf. Introduction). Thus, the probability of shared input is high within a limit of $3000 \mu$ and very low outside this distance.

The strength of shared input correlation did not show significant variation across this distance (Fig. 4). This is in keeping with 
A

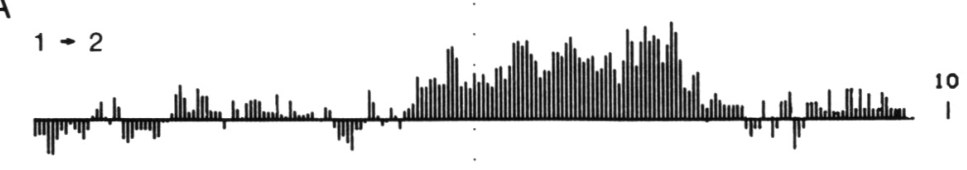

B

$1 \rightarrow 3$
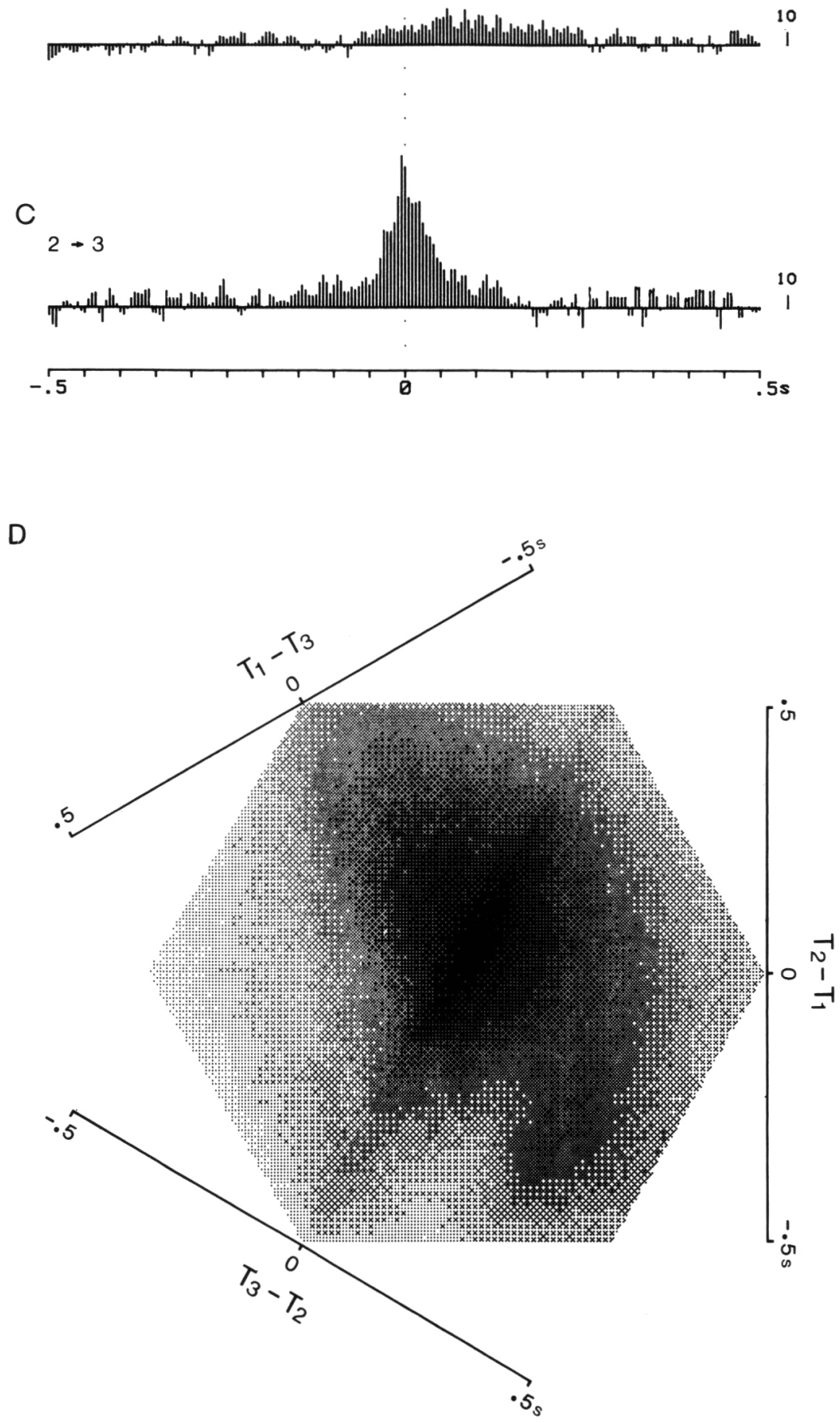

Figure 8 - Shared input to three neurons recorded simultaneously.

A-C: $5 \mathrm{~ms}$ bin difference correlograms of 3 possible cell pairings (1-2, $1-3$ and $2-3)$.

$D$. Joint impulse scatter diagram of the above three cells using hexagonal-shaped bins of 20 ms width. Bin count ranges from 14 to 1096 and is represented by $a$ grey density scale of 10 divisions. 
the nature of shared input, which would involve branching of axons from a remote source. A possible exception is noted when each member of a cell pair is recorded from the same electrode. In this situation, the maximum between cells is about $250 \mu$ (Stoney et al., 1968; Rosenthal, 1972). Thus, many of these cells should be in the same functional column. In our data, all pairs recorded from the same electrode were indeed coupled to the same joints, as measured by the independent means of ICMS and passive sensory stimulation.

Interestingly, the average width of one of the nested forelimb zones in motor cortex is about $750 \mu$ (Wong et al., 1978; Kwan et al., 1978; Murphy et al., 1978). The minimum distance between the shoulder zone and the finger zone is about $1500 \mu$ (ibid). Thus, on distance considerations alone, the possibility exists for input from a common source to be distributed to nonadjacent forelimb joints. In fact, the data did not confirm this possibility. The probability of shared input between cells within columns representing adjacent joints was approximately equal to that for cell pairs within the same column (Table 2). In contrast, the probability of shared input between cells in nonadjacent joint zones was very low. We conclude that shared input is concentrated among columns in the same and adjacent joint controlling zones, as well as within a single column.

Synaptic interaction and shared input are quite sharply distinguished by the temporal properties of the correlation plots in this paradigm. The duration of significant peaks in difference correlograms is less than $10 \mathrm{~ms}$ in the case of synaptic interaction, whereas durations of $80-480 \mathrm{~ms}$ are observed in the shared input case (Fig. 6). Similarly, the peaks (or valleys) in the correlograms are invariably centered within $20 \mathrm{~ms}$ of the origin in the case of synaptic interaction (Fig. 3), while a much broader range of peak loci are seen in instances of shared input (Fig. 6B). These temporal features leave little ambiguity in assessing the presence of synaptic interaction, shared input or both in an individual cell pair. Similar temporal distinctions have been noted in other contexts (Perkel et al., 1967; Moore et al., 1970; Dickson and Gerstein, 1974; Michalski et al., 1983; Mastronade, 1983).

In view of the nested organization of the forelimb area of primate motor cortex (Murphy et al., 1978), we were interested to determine whether any preferred orientation of correlated pairs could be demonstrated. The data of Figure 7 show that no preferred direction is present. In particular, the polar frequency plot of Figure 7B indicates that shared input correlation is extended in all directions radial to any particular cell or column. This observation is compatible with a nested organization. On a functional level, such a multidirectional divergence of input from a common source would afford potential interaction among the maximum number of columns within the joint controlling zones. This in turn would allow optimum flexibility in movement control, and as well add a dimension of functional redundancy. The latter would be particularly useful in reducing errors in situations of cellular dysfunction due to pathology or other local factors (Sabah and Murphy, 1971; Von Neumann, 1956).

Anatomic studies show that axons reaching motor cortex from various sources typically have multiple rather than single branches (cf. Introduction). Sampling limitations would be expected to render quite difficult the demonstration of shared input interactions among more than 2 neurons. We were able to discern such interactions in 4 of 41 cases in which spike trains were recorded simultaneously from 3 or more neurons. The presence of such divergent transmission from a common source (Fig. 8) supports the anatomic observation of branching axons. It also adds to evidence derived from spatial analysis (Fig. 7, Table 2) that separate columns of cells in motor cortex are activated by input from a common source during the reaching movement. This arrangement would allow particular multijoint movements and sequences of movements to be selected and coordinated by such common sources.

The actual sources of shared inputs demonstrated in these studies could include either or both corticocortical and thalamocortical systems. Anatomic and physiologic details of corticocortical projection systems to motor cortex are gradually accumulating, with known projection systems from parietal, prefrontal and occipital regions (Humphrey, 1983). Deep, midline extensions of the reticular activating system would be expected to project widely to all areas of cerebral cortex, in keeping with an arousal function. Specific input systems to motor cortex from VL thalamus have been elegantly studied by Strick (1976a and $b$ ). Further anatomic studies will undoubtedly be of major importance in developing an understanding of how the columnar interactions which we have demonstrated in motor cortex are activated to produce voluntary forelimb movements.

\section{ACKNOWLEDGEMENTS}

This research was supported by MRC of Canada. We thank $\mathrm{H}$. Nguyen Huu for programming assistance.

\section{REFERENCES}

Allum JHJ, Hepp RMC and Gysin R (1982) Cross correlation analysis of interneuronal connectivity in the motor cortex of the monkey. Brain Research, 231: 325-334.

Asanuma H and Rosen I (1973) Spread of mono- and polysynaptic connections within cat motor cortex. Exp Brain Res 16: 507-520.

Dickson JW and Gerstein GL (1974) Interactions between neurons in auditory cortex of the cat. J Neurophysiol 37: 1239-1261.

Fetz EE and Cheney PD (1978) Muscle fields of primate corticomotorneuronal cells. J Physiol (Paris) 74: 239-245.

Gatter KC and Powell TPS (1978) The intrinsic connections of the cortex of area 4 of the monkey. Brain 101: 513-542.

Harnett DL (1975). Introduction to statistical methods. 2nd Ed. AddisonWesley, Mass., pp 529-532.

Humphrey DR (1979) On the cortical control of visually directed reaching: Contributions by non-precentral motor areas. In: Posture and Movement, Talbot, RE and Humphrey, DR (eds). Raven Press, N.Y.

Jones EG (1983) Thalamic basis for column-like input to monkey somatic sensory and motor cortex. In: Somatosensory Integration in the Thalamus. G Macchi et al (eds). Elsevier, Amsterdam, pp 309-336.

Jones EG, Coulter JD and Hendry SHC (1978) Intracortical connectivity of architectonic fields in the somatic sensory, motor and parietal cortex of monkeys. J Comp Neur 181: 291-348.

Jones EG, Coulter JD and Wise SP (1979) Commissural columns in the sensory-motor cortex of monkeys. J Comp Neur 188: 113-136.

Jones EG and Wise SP (1977) Size, laminar and columnar distribution of efferent cells in the sensory motor cortex of monkeys. J Comp Neurol 175: 391-438.

Kelly JS and Renaud LP (1974) Physiologic identification of inhibitory interneurons in the feline pericruciate cortex. Neuropharmacology 13: 463-474.

Knox CK (1981) Detection of neuronal interactions using correlation analysis. TINS 4: 222-225.

Kwan HC, MacKay WA, Murphy JT and Wong YC(1978) Organization of precentral cortex in awake primates. II Motor outputs. J Neurophysiol 41: 1120-1131. 
Kwan HC, MacKay WA, Murphy JT and Wong YC (1980) Recordings of simultaneous spike trains by two independent electrodes in awake primates. Physiol and Behav 25: 321-322.

Kwan HC, MacKay WA, Murphy JT and Wong YC (1981) Distribution of responses to visual cues for movement in precentral cortex of awake primates. Neurosci Lett 24: 123-128.

Lamarre Y, Spidaliri G and Lund JP (1981) Patterns of muscular and motor cortical activity during a simple arm movement in the monkey. Am J Physiol Pharmacol 59: 748-756.

Mastronade DN (1983) Correlated firing of cat retinal ganglion cells. I Spontaneously active inputs to $x$-and $y$-cells. J Neurophysiol 49: 303-324.

Matsumura M (1979) Intracellular synaptic potentials of primate motor cortex neurons during voluntary movement. Brain Research 163: 33-48.

Michalski A, Gerstein GL, Garkowska J and Tarnecki R (1983) Interactions between cat striate cortex neurons. Exptl Brain Res 51: 97-107.

Moore GP, Segundo JP, Perkel DH and Levitan H (1970) Statistical signs of synaptic interaction in neurons. Biophys J 10: 876-900.

Murphy JT, Kwan HC, MacKay WA and Wong YC (1978) Spatial organization of precentral cortex in awake primates. III. Inputoutput coupling. J Neurophysiol 41: 1132-1139.

Murphy JT, Kwan HC and Wong YC (1985a) Cross correlation studies in primate motor cortex. II. Event related correlation. Can J Neurol Sci

Murphy JT, Kwan HC and Wong YC (1985b). Sequential activation of neurons in primate motor cortex during unrestrained forelimb movement. $\mathrm{J}$ Neurophysiology, in press.

Murphy JT, Kwan HC, MacKay WA and Wong TC (1982a) Activity of primate precentral neurons during voluntary movements triggered by visual signals. Brain Research 236: 429-449.

Murphy JT, Kwan HC, MacKay WA and Wong YC (1982b) Precentral unit activity correlated with angular components of a compound movement. Brain Research 246: 141-145.

Perkel DH, Gerstein GL and Moore GP (1967) Neuronal spike trains and stochastic point processes. II. Simultaneous spike train. Biophys J 7: $419-440$.

Perkel DH, Gerstein GL, Smith MS and Tatton WG (1975) Nerve- impulse patterns: A quantitative display technique for three neurons. Brain Research 100: 271-296.

Phillips CG (1981) Microarchitecture of motor cortex of primates. In: Progress in Anatomy, Vol 1. Harrison RJ and Holmes RL (eds). Cambridge University Press, Cambridge, pp. 61-94.

Rosenthal F (1972) Extracellular potential fields of single PT-neurons. Brain Res 36: 251-263.

Sabah NH and Murphy JT (1971) A superposition model of the spontaneous synaptic excitation of cerebellar Purkinje cells. Biophys J 11: 414-428.

Sears TA and Stagg D. Short-term synchronization of intercostal motorneuron activity. J Physiol (London) 263: 357.381.

Soechting JF (1984) Effect of target size on spatial and temporal characteristics of a pointing movement in man. Expt Brain Res 54: 121-132.

Stoney SD, Thompson WD and Asanuma H (1968) Excitation of pyramidal tract cells by intracortical microstimulation: effective extent of stimulating current. J Neurophysiol 31: 659-669.

Strick PL (1976a) Anatomical analysis of ventrolateral thalamic input to primate motor cortex. J Neurophysiol 39: 1020-1031.

Strick PL (1976b) Activity of ventrolateral thalamic neurons during arm movement. J Neurophysiol 39: 1032-1044.

Szentagothai J (1978) The neuron network of the cerebral cortex. A functional interpretation. Proc R Soc Lond Ser B 201: 219-248.

Tatton WG and Sokolove PG (1975) Analysis of postural motoneuron activity in crayfish abdomen. 11. Co-ordination by excitatory and inhibitory connections between motoneurons. J Neurophysiol 38: 332-346.

Von Neumann J (1956) Probabilistic logics and the synthesis of reliable organisms from unreliable components. In: Automata Studies, Shannon, CE and McCarthy, J (eds), Princeton University Press, Princeton, pp 43-98.

Wong YC, Kwan HC, Mackay WA and Murphy JT (1978) Spatial organization of precentral cortex in awake primates. I. Somatosensory inputs. J Neurophysiol 41: 1107-1119.

Wong YC, Kwan HC and Murphy JT (1980) Temporal characteristics of torque-triggered neuronal responses in primate precentral cortex. Can J Physiol Pharmacol 58: 778-786. 